\title{
METODOLOGÍA DE ANÁLISIS MULTICRITERIO APLICACIÓN AL CRECIMIENTO SOSTENIBLE EN LA UNIÓN EUROPEA
}

\author{
María Victoria Hermenegildo Chávez* \\ maryvher@gmail.com \\ Yolanda Rueda Osuna ${ }^{* *}$ \\ yruedaosuna@gmail.com
}

\begin{abstract}
RESUMEN
Este trabajo tiene como objetivo fundamental mostrar la metodología de Análisis Multicriterio PROMETHEE, en la que se realiza una clasificación de alternativas y entre éstas se elige a la mejor, aplicando un conjunto de criterios que se encuentran en conflicto entre sí.

Se realizará un estudio de ordenamiento de países de la Unión Europea determinando el país que lidera y avanza hacia el desarrollo sostenible (el que domina a los demás), haciendo uso, a la vez de potentes aplicaciones informáticas para lograr este objetivo. Los datos han sido obtenidos de EUROSTAT del año 2012, dado que publica resultados cada dos años.
\end{abstract}

Palabras claves: Decisión multicriterio, clasificación, preferencia, indiferencia, consistencia.

\section{ABSTRACT}

This work have as main objective to show the methodology of PROMETHEE Multicriteria Analysis, performing a ranking of alternatives and between them we will choose the best, applying a set of criteria that are in conflict with each other.

We will do a study of ranking of countries of Europe Union to find what country leads and moves towards to sustainable development (country that dominates at the other countries), and we will use computer tools powerfull in order to achieve this goal. The data have been obtained of EUROSTAT 2012, because the results are published each two years.

Keywords: Multicriterio decision, ranking, preference, indifference, consistency.

\footnotetext{
* Máster en Investigación en Gestión por la Universidad Pablo de Olavide de Sevilla (España), Magíster en Ciencias de la Computación por la Universidad de Cantabria (España), Estudios de Maestría en Ciencias por la Universidad Nacional de Ingeniería (UNI), Licenciada en Matemáticas por la Universidad Nacional de Trujillo (UNT), Docente Auxiliar de la Facultad de Ciencias Administrativas (FCA) de la Universidad Nacional Mayor de San Marcos (UNMSM), Docente de la Unidad de Postgrado de la FCA (UNMSM).

***áster en Gestión Estratégica y Negocios Internacionales por la Universidad de Sevilla (España). Licenciada en Investigación y Técnicas de Mercado por la Universidad de Sevilla. Diplomada en Estadística por la Universidad de Sevilla. Funcionaria de la Consejería de Educación de la Junta de Andalucía (España).
} 


\section{INTRODUCCIÓN}

En la última década, se han incrementado el número de publicaciones sobre decisión multicriterio en donde se proponen interesantes métodos, pero la ventaja de los métodos PROMETHEE es que requieren información adicional muy clara y precisa (Fernández, 2002).

La técnica multicriterio PROMETHEE ha sido aplicada a muchas áreas de investigación: en el sector ambiental, en recursos humanos y turísticos (Franco-Maass et al., 2013), en selección de proyectos, estructuras industriales de la economía (Sala, 2000), oportunidades de comercialización, políticas sociales y ambientales (Pujol, 2002), en mercados financieros (Fernández, et al., 2009), investigación del transporte, problemas de ubicación (Guerrero, 2007) y muchas áreas más, de allí la importancia de mostrar cómo funciona esta técnica y para ello lo aplicamos a un caso real para ilustrar la metodología, haciendo uso de los software Decision Lab y Expert Choice. Los resultados obtenidos permiten observar las diferencias entre cada una de las alternativas estudiadas, y la técnica multicriterio indica qué aspectos se deben mejorar para que una alternativa alcance mayor puntuación en la clasificación.

Se han escogido 23 países de la Unión Europea (UE), y se realizará una ordenación usando Indicadores de Desarrollo Sostenibles (IDS), elaborados por la Organización de las Naciones Unidas (ONU) en 1995 y recopilados por la UE, mostrando qué indicadores debe reforzar cada país para seguir avanzando hacia el desarrollo sostenible en estos momentos de crisis económica y financiera.

Por simplicidad se mostrará en algún punto solo los resultados de la alternativa que lidera a las demás y de la alternativa que es dominada por los demás. Asimismo, la información no reciente del caso podría sesgar los resultados obtenidos al aplicar esta metodología.

\section{MÉTODOS}

El análisis de decisión multicriterio se define como un conjunto de técnicas orientadas a asistir en procesos de decisión. Se basa en la ponderación y comparación de variables que van a influir de manera positiva o negativa sobre la actividad objeto de decisión. Estos métodos sirven para hallar soluciones posibles no necesariamente óptimas.
Existen dos o más alternativas de solución y dos o más criterios que permiten evaluar las alternativas, asimismo los objetivos indican direcciones de perfeccionamiento o niveles de satisfacción de los atributos.

Como principales métodos de análisis de decisión multicriterio se consideran: Ponderación Lineal (Scoring), Utilidad Multiatributo (MAUT), y, Relaciones de Superación dentro de la cual están los métodos de la Escuela Francesa: ELECTRE (Elimination et Choix Traduisant la Realité) (Romero, 1996) y PROMETHEE (Preference Ranking Organization Method for Enrichment Evaluation), así como también el Proceso de Análisis Jerárquico (AHP, siglas tomadas de su nombre en inglés: The Analytic Hierarchy Process) creado por el Matemático Thomas L. Saaty (1980).

Los métodos de Relaciones de Superación se basan en comparaciones binarias de alternativas, criterio por criterio.

\section{MÉTODO DE ANÁLISIS MULTICRITERIO PROMETHEE}

El método PROMETHEE para análisis multicriterio que se utiliza en este trabajo, parte de la base de que el decisor debe establecer la importancia relativa de cada uno de los criterios, para luego definir una estructura de preferencias entre las alternativas.

Se ofrecen dos posibilidades para resolver el problema de ordenamiento: obtener un pre orden parcial (PROMETHEE I) u obtener un pre orden completo (PROMETHEE II), ambos sobre el conjunto de alternativas factibles (Fernández, 2002). Calculamos para ello los pesos de cada uno de los criterios mediante el software Expert Choice; se definen las funciones de preferencia, y la información se ingresa al Decision Lab.

En el proceso de asignación de la importancia entre criterios se obtiene un índice de inconsistencia: si es menor que 0,1 se considera aceptable la asignación de pesos; por tanto, existirá consistencia entre las puntuaciones asignadas a las variables.

\section{Funciones de preferencia o indiferencia}

Los criterios generalizados son funciones de preferencia que se utilizan en la etapa de modelación de las preferencias, y evitan la subjetividad a la hora de delimitar los umbrales de preferencia y/o de indiferencia que se requieran para cada criterio (Fernández \& Escribano, 2006). 
Esta fase requiere que a cada criterio se le asocie una función mediante la cual se valora la preferencia de una alternativa con respecto a otra, como función de la diferencia entre evaluaciones. Con este propósito están implementadas en el software Decision Lab seis tipos de funciones: Usual (Normal), Linear (Lineal), Level (Escalonado), U-Shape (en forma de U), V-Shape (en forma de V) y Gaussian (Gaussiana), que nos sirven para establecer preferencias en función de tres parámetros: q, umbral que define el área de indiferencia; $p$, umbral que define el área de preferencia estricta; y d, parámetro que relaciona los valores de p y q. Para asignar las funciones a los criterios se consideran las medias y desviaciones estándar de los datos recogidos, y la aplicación del sentido común y experiencia en otros casos.

A continuación se presentan los seis tipos de criterios generalizados, su definición y gráfica:

\section{Criterio Usual}

Figura $\mathbf{N}^{\circ} 1$

$$
P(d)=\left\{\begin{array}{llcc}
0 & \text { si } & d \leq 0 & \text { (Indiferencia) } \\
1 & \text { si } d>0 & \text { (Preferencia estricta) }
\end{array}\right.
$$

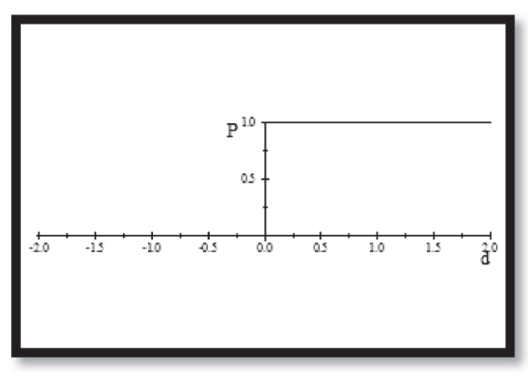

\section{Criterio U-shape}

Figura $\mathrm{N}^{\circ} 2$

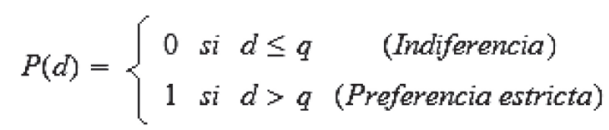

Fijar el parámetro q, en la Figura $\mathrm{N}^{\circ} 2$ donde $\mathrm{q}=2$.



\section{Criterio V-shape}

Figura $\mathrm{N}^{\circ} 3$

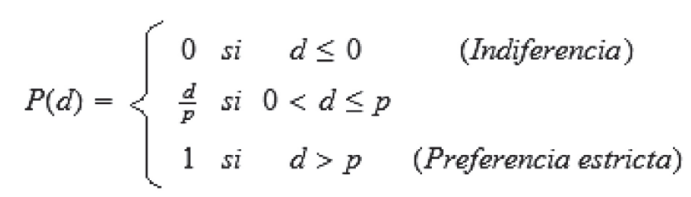

Parámetro $\mathrm{p}$ a fijar. En la Figura $\mathrm{N}^{\circ} 3$ donde $\mathrm{p}=2$.

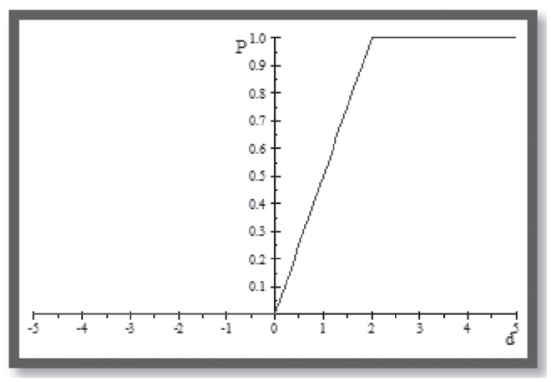

\section{Criterio Level}

\section{Figura $\mathbf{N}^{\circ} 4$}

$$
P(d)=\left\{\begin{array}{cccc}
0 & \text { si } & d \leq q & \text { (Indiferencia) } \\
\frac{1}{2} & \text { si } & q<d \leq p & \\
1 & \text { si } & d>p & \text { (Preferencia estricta) }
\end{array}\right.
$$

Parámetros a fijar q y p; en la Figura $\mathrm{N}^{\circ} 4$, donde $\mathrm{q}=1$ y $\mathrm{p}=3$.

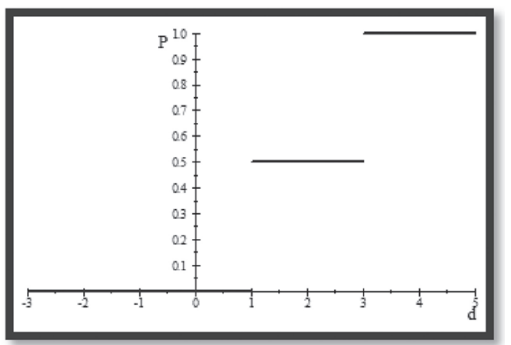

\section{Criterio Linear}

\section{Figura $\mathrm{N}^{\circ} 5$}

$$
P(d)=\left\{\begin{array}{cccc}
0 & \text { si } & d \leq q & \\
\frac{d-q}{p-q} & \text { si } & q<d \leq p \\
1 & \text { si } & d>p & \text { (Indiferencia) }
\end{array}\right.
$$

Parámetros a fijar q y p; en la Figura $\mathrm{N}^{\circ} 5$, donde $\mathrm{q}=1$ y $\mathrm{p}=3$.






\section{Criterio Gaussian}

\section{Figura $\mathrm{N}^{\circ} 6$}

$$
P(d)=\left\{\begin{array}{cc}
0 & \text { si } d \leq 0 \\
1-e^{-\frac{d^{2}}{2 s^{2}}} & \text { si } d>0
\end{array}\right.
$$

Donde $S$ es la desviación estándar; en la Figura $\mathrm{N}^{\circ} 6, \mathrm{~S}=2$.

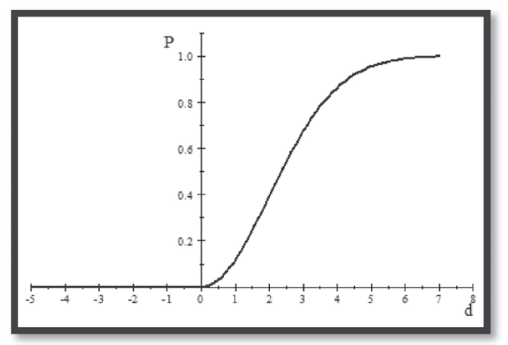

\section{El PLANO GAIA}

El proceso GAIA (Geometrical Analisis for Interactive AID) es complementario a la Metodología PROMETHEE y es una poderosa herramienta de decisión cualitativa (Mareschal and Brans, 1988; Férnandez, 2002 ). El plano GAIA ofrece una descripción gráfica del problema de decisión, enfatizando los conflictos existentes entre los criterios y el impacto de los pesos en la decisión final, y es más descriptivo que los métodos PROMETHEE (Fernández, 2002, p.6).

\section{DESCRIPCIÓN DEL CASO: DESARROLLO SOSTENIBLE EN LA UNIÓN EUROPEA}

La Comisión Mundial de la Organización de las Naciones Unidas para el Desarrollo y Medio Ambiente en 1987, publicó el denominado Informe Brundtland, donde la primera ministra Noruega, Gro Harlem Brundtland califica al desarrollo sostenible de un país como aquel desarrollo que es capaz de satisfacer las necesidades actuales sin comprometer los recursos y posibilidades de las futuras generaciones.

En 1995, la Organización de las Naciones Unidas (ONU) propuso elaborar un conjunto de 134 indicadores sobre desarrollo sostenible, a los que tuvieran acceso los encargados de la toma de decisiones a nivel internacional. Cada país debía seleccionar aquellos indicadores que sean pertinentes para sus metas, prioridades y objetivos nacionales, con la finalidad de mejorar la calidad de vida y el bienestar de las generaciones presentes y futuras, vinculando el desarrollo económico, la protección del medio ambiente y la justicia social. Se crea así en el 2006 la denominada Estrategia de Desarrollo Sostenible (EDS) que describe cómo debe la UE afrontar los retos del desarrollo sostenible de una forma más eficaz (Eurostat, 2012). En el 2007, la Comisión Europea (EC) determinó y actualizó un grupo de indicadores (European Commission, 2006) que se aprobaron en el 2005.

El Sustanaible Development International (SDI) plantea diez temas, cada uno de los cuales contiene una serie de aspectos socio económicos y medio ambientales que se relacionan con cada uno de los siete desafíos recogidos en detalle por la Estrategia Europea de Desarrollo Sostenible (European Commission, 2006).

Con la finalidad de examinar si se ha logrado un progreso hacia el desarrollo sostenible en términos de objetivos y metas, la Comisión Europea realiza una recopilación de un conjunto de indicadores, y realiza un informe de seguimiento cada dos años (Eurostat, 2012; Comisión Europea, 2012). Hasta la actualidad, se han publicado un total de cuatro informes de seguimiento (en el 2005, 2007, 2009 y 2012, uno cada año) que exponen los principales avances y las deficiencias observadas en este tema.

En estos informes de seguimiento, la UE determina cuáles son los temas y los indicadores considerados como principales (véase Cuadro $\mathrm{N}^{\circ} 1$ ) del total de 134 enunciados por la ONU.

- Alternativas. Las alternativas u opciones del problema multicriterio serán 23 de los 28 países de la Unión Europea (los países elegidos se muestran en el Cuadro $\mathrm{N}^{\circ} 7$ ). Los países excluidos son Bulgaria, Malta, Polonia y Rumanía porque EUROSTAT carece de datos fiables sobre estos países, relacionados con los indicadores de desarrollo en estudio.

- Criterios. La UE en sus Informes de Seguimiento sobre la EDS determina un indicador principal para cada uno de los temas que la ONU considera como importantes para avanzar hacia el Desarrollo Sostenible (ver Cuadro $\mathrm{N}^{\circ} 1$ ). Hemos elegido estos indicadores como criterios para realizar nuestro análisis, a excepción del indicador Buena Gobernanza, ya que carece de indicador principal (observe Cuadro $\mathrm{N}^{\circ} 2$ ), y además se muestra la notación usada, las unidades y el objetivo de cada indicador. 
Cuadro $\mathrm{N}^{\circ} 1$. Indicadores económicos principales utilizados por la UE

\begin{tabular}{|l|l|}
\hline \multicolumn{1}{|c|}{ TEMA } & \multicolumn{1}{|c|}{ INDICADOR PRINCIPAL } \\
\hline Desarrollo Socioeconómico & Crecimiento PBI per cápita \\
\hline Producción y Consumo Sostenible & Productividad de los recursos \\
\hline Inclusión Social & Tasa de riesgo de exclusión social \\
\hline Cambios Demográficos & Tasa de empleo de trabajadores mayores \\
\hline Salud Pública & Esperanza de vida sana \\
\hline Cambios Climáticos y Energía & $\begin{array}{l}\text { Porcentaje de energía renovable en el consumo final } \\
\text { bruto de energía }\end{array}$ \\
\hline Transporte Sostenible & $\begin{array}{l}\text { Consumo de energía del transporte en relación con } \\
\text { el PBl }\end{array}$ \\
\hline Recursos Naturales & Porcentaje de deforestación \\
\hline Alianzas Internacionales & Ayuda oficial al desarrollo como porcentaje del PBI \\
\hline Buena Gobernanza & [Sin indicador principal] \\
\hline
\end{tabular}

Fuente: Eurostat, 2012.

En su mayoría corresponde con datos de los años 2011, 2010 o 2009, así como de años anteriores debido a la falta de información de la UE

\section{OBJETIVO PRINCIPAL}

Mostrar la aplicación de la Metodología Multicriterio a un caso real. Para lograr este objetivo se eligieron 23 países de la UE (alternativas), y 9 indicadores de sostenibilidad (criterios). Los objetivos específicos del caso se indican en el Cuadro $\mathrm{N}^{\circ} 2$.

\section{Cuadro $\mathrm{N}^{\circ} 2$ Objetivos específicos}

\begin{tabular}{|l|c|c|c|}
\hline \multicolumn{1}{|c|}{ INDICADOR PRINCIPAL } & NOTACIÓN & UNIDADES & OBJETIVO \\
\hline Crecimiento Producto Bruto Interno (PBI) per Cápita & Crec_PBI & Euros & Maximizar \\
\hline Productividad de los Recursos & Producti & Euros/kg. & Maximizar \\
\hline Tasa de Riesgo de Exclusión Social & Tasa_Rie & Porcentaje & Minimizar \\
\hline Tasa de Empleo de Trabajadores Mayores (55 a 64 años) & Tasa_Emp & Porcentaje & Maximizar \\
\hline Esperanza de Vida Sana a partir de los 65 años & Esper_Vi & Años & Maximizar \\
\hline $\begin{array}{l}\text { Porcentaje de Energía Renovable en el Consumo Final } \\
\text { Bruto de Energía }\end{array}$ & EnergiaR & Porcentaje & Maximizar \\
\hline $\begin{array}{l}\text { Consumo de Energía del Transporte en relación con el } \\
\text { PBI }\end{array}$ & ConsumoE & No. índice & Minimizar \\
\hline Porcentaje de Deforestación & IndiceFo & Porcentaje & Minimizar \\
\hline Ayuda oficial al desarrollo como porcentaje del PBI & AyudlntP & Porcentaje & Maximizar \\
\hline
\end{tabular}




\section{ASOCIACIÓN DE FUNCIONES DE PREFERENCIA O INDIFERENCIA A LOS CRITERIOS}

Para definir los umbrales tanto de preferencia como de indiferencia se ha considerado la media y la desviación estándar de cada criterio que se muestran en el Cuadro $\mathrm{N}^{\circ} 6$, y los valores mínimo y máximo de cada criterio se observan en la Cuadro $\mathrm{N}^{\circ} 3$ :

Cuadro $\mathrm{N}^{\circ} 3$

\begin{tabular}{|c|c|c|}
\hline NOTACIÓN & Valor mínimo & Valor máximo \\
\hline Crec_PBI & 6400 & 65099 \\
\hline Producti & 2.54 & 1038.8 \\
\hline Tasa_Rie & 14.4 & 38.1 \\
\hline Tasa_Emp & 31.2 & 72.3 \\
\hline Esper_Vi & 3.05 & 14.8 \\
\hline EnergiaR & 0 & 47.3 \\
\hline ConsumoE & 88.1 & 121.9 \\
\hline IndiceFo & 6.2 & 51.1 \\
\hline AyudIntP & 0.06 & 1.09 \\
\hline
\end{tabular}

\section{Crecimiento del Producto Bruto Interno (PBI) per Cápita}

Se usará la función Linear que nos indica que si la desviación del crecimiento del Producto Bruto Interno per cápita es menor de $€ 4.580$, nos es indiferente. Si el crecimiento está entre $€ 4.580$ y $€ 9.160$ asumimos con una preferencia débil que es sostenible; y si la diferencia es mayor a $€ 9.160$, con alta preferencia podemos decir que en la actualidad un país es sostenible o no, y si no lo es, no será sostenible ni a corto ni a mediano plazo.

\section{Productividad de los recursos}

También se utiliza la función Linear. Para la sostenibilidad de un país: si la diferencia en la productividad de los recursos es de $€ 80$ por kilogramo, nos es indiferente; si está entre $€ 80$ y $€ 180$ por kilogramo, asumimos que un país es sostenible con un nivel de preferencia débil; y si la diferencia es mayor que $€ 180$ por kilogramo, con alta preferencia podemos afirmar que en la actualidad un país es sostenible o no, y si no lo es, no será sostenible ni a corto ni a mediano plazo.

\section{Tasa de riesgo de exclusión social}

Se trabaja con la función V-shape. Con una desviación mayor a 3\% de tasa de riesgo de exclusión social y con alta preferencia se decidirá si un país es sostenible o no.

\section{Tasa de empleo de los trabajadores mayores}

Se usa la función Gaussian. La diferencia de $3,5 \%$ en la tasa de empleo de los trabajadores mayores nos es indiferente para decidir si un país es sostenible o no.

\section{Esperanza de vida sana a partir de los 65 años}

Se trabaja con la función Linear. Para la sostenibilidad de un país, si la diferencia en el indicador de la esperanza de vida sana a partir de los 65 años es de 1,2 años, no es indiferente. Si está entre 1,2 y 2,4 años asumimos con un nivel de preferencia débil que un país es sostenible. Y si la diferencia es mayor que 2,4 años con alta preferencia podemos decir que en la actualidad un país es sostenible o no, y si no es sostenible, nos podría indicar que el mayor porcentaje de personas no tendrían una vida saludable a partir de los 65 años en ese país.

6. Porcentaje de la energía renovable en el consumo final bruto de energía

Se usará la función Gaussian. La diferencia de $4 \%$ de energía renovable en el consumo final bruto de energía nos es indiferente para decidir si el país es sostenible o no; a mayor diferencia es muy importante porque los países deberían fomentar la energía renovable para su competitividad y para que se incremente la sostenibilidad del país.

\section{Consumo de energía de transporte en relación} con el PBI

Se usará la función Gaussian. La diferencia de 2,6 en el índice de consumo de energía de transporte en relación con el PBI, nos es indiferente para decidir si un país es sostenible o no.

\section{Porcentaje de deforestación}

Se usará la función Gaussian. La desviación de $4 \%$ en el indicador de deforestación no será una diferencia significativa para decidir si el país es sostenible o no.

\section{Ayuda oficial al desarrollo como porcentaje del PBI}

Se trabaja con la función U-shape. Para decidir si un país es sostenible: una desviación de $0,16 \%$ es indiferente a que un país reciba la ayuda oficial al desarrollo o no, recordando que éste era el indicador de menos importancia.

Una observación importante en la definición de funciones, es que se podría haber usado la función Level en los indicadores PBI per cápita y productividad de recursos pero, para dar mayor flexibilidad al indicador, se ha usado la función Linear. 


\section{RESULTADOS Y DISCUSIÓN}

Se procede a la construcción de los pesos asociados a cada criterio, para lo cual se utiliza la técnica AHP y luego lo implementamos en el método PROMETHEE.

Considerando la variable de menor importancia ayuda oficial al desarrollo como porcentaje del PBI, denotada por AyudIntP, e ingresando la relevancia de cada criterio, ver Cuadro $\mathrm{N}^{\circ} 4$ :

Cuadro $\mathrm{N}^{\circ} 4$

\begin{tabular}{|l|c|}
\hline & Value \\
\hline Crec_PBI & $\mathbf{5 . 0 0 0 0 0}$ \\
\hline Producti & 5.00000 \\
\hline Tasa_Rie & $\mathbf{3 . 0 0 0 0 0}$ \\
\hline Tasa_Emp & $\mathbf{3 . 0 0 0 0 0}$ \\
\hline Esper_Vi & $\mathbf{3 . 0 0 0 0 0}$ \\
\hline EnergiaR & $\mathbf{4 . 0 0 0 0 0}$ \\
\hline ConsumoE & $\mathbf{4 . 0 0 0 0 0}$ \\
\hline IndiceFo & 2.00000 \\
\hline AyudlntP & $\mathbf{1 . 0 0 0 0 0}$ \\
\hline Total & 30.00000 \\
\hline
\end{tabular}

Fuente: Elaboración propia

Asimismo, se construye la matriz de comparación por pares de alternativas en el Expert Choice (Cuadro $\mathrm{N}^{\circ} 5$ ):

\section{Cuadro $N^{\circ} 5$}

\begin{tabular}{|c|c|c|c|c|c|c|c|c|}
\hline IRest Fitl & Producti & Tasa Rie & Iasa Emp & Esper $V_{i}$ & EnergiaR & ConsumoE & Indicefo & ApudlntP \\
\hline Crec PBI & 1.0 & 1.7 & 1.7 & 1.7 & 1.3 & 1.3 & 2.5 & +5.0 \\
\hline Producti & & 1.7 & 1.7 & 1.7 & 1.3 & 1.3 & 2.5 & 5.0 \\
\hline Tasa Rie & & & 1.0 & 1.0 & 1.3 & 1.3 & 1.5 & 3.0 \\
\hline Tasa Emp & & & & 1.0 & 1.3 & 1.3 & 1.5 & 3.0 \\
\hline Esper $V_{i}$ & & & & & 1.3 & 1.3 & 1.5 & 3.0 \\
\hline EnergiaR & & & & & & 1.0 & 2.0 & 4.0 \\
\hline ConsumoE & & & & & & & 2.0 & 4.0 \\
\hline IndiceFo & & & & & & & & 2.0 \\
\hline
\end{tabular}

Fuente: Elaboración propia

Calculando los pesos con el software Expert Choice (ver Figura $\mathrm{N}^{\circ} 7$ ):

\section{Figura $\mathbf{N}^{\circ} 7$}

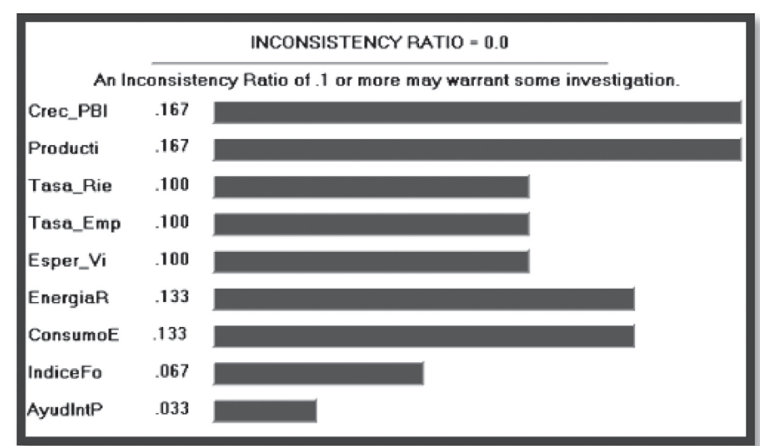

Fuente: Elaboración propia

Una vez que se ha definido el objetivo de cada criterio maximizar o minimizar que se muestran en el Cuadro $\mathrm{N}^{\circ} 2$, se ingresa la información en el software Decision Lab (ver Cuadro $\mathrm{N}^{\circ} 6$ ), donde se ha omitido la matriz de decisión, que contiene la información correspondiente a las alternativas con respecto a cada criterio:

\section{Cuadro $N^{\circ} 6$}

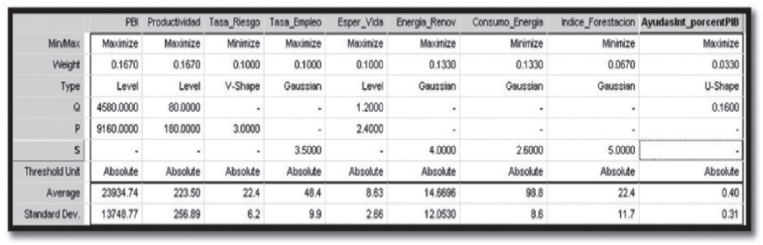

Fuente: Elaboración propia

\section{Resultados según PROMETHEE I}

\section{Ranking parcial}

Analizando este ranking (resultados según el software Decision Lab recogidos en el Cuadro $\mathrm{N}^{\circ} 7$ ), Suecia es el candidato más fuerte porque presenta $\varnothing+=0,68$ y el menos débil $\emptyset-=0,07$; por lo tanto, es el país con mayor crecimiento sostenible porque Suecia domina o se sobrepone al resto de los países.

Hungría es el candidato menos fuerte porque presenta $\emptyset+=0,05$, y el más débil con

$\emptyset-=0,50$, es el país que menos avanza hacia el desarrollo sostenible.

Cuadro $\mathrm{N}^{\circ} 7$

\begin{tabular}{|l|c|c|c|}
\hline \multicolumn{1}{|c|}{ País } & PROMETHEE 1 & PROMETHEE 2 & \\
\hline & Flujo de entrada $\varnothing+$ & Flujo de salida $\emptyset-$ & Flujo neto $\emptyset$ \\
\hline Suecia & 0,70 & 0,07 & 0,64 \\
\hline Finlandia & 0,52 & 0,12 & 0,40 \\
\hline Holanda & 0,43 & 0,19 & 0,23 \\
\hline Dinamarca & 0,53 & 0,20 & 0,33 \\
\hline Alemania & 0,53 & 0,20 & 0,35 \\
\hline Reino Unido & 0,53 & 0,23 & 0,30 \\
\hline Francia & 0,49 & 0,25 & 0,24 \\
\hline Irlanda & 0,42 & 0,26 & 0,16 \\
\hline Austria & 0,41 & 0,27 & 0,14 \\
\hline España & 0,43 & 0,31 & 0,12 \\
\hline Bélgica & 0,27 & 0,37 & $-0,10$ \\
\hline Chipre & 0,27 & 0,37 & $-0,11$ \\
\hline Estonia & 0,32 & 0,37 & $-0,05$ \\
\hline Italia & 0,35 & 0,38 & $-0,03$ \\
\hline Luxemburgo & 0,38 & 0,39 & $-0,01$ \\
\hline Lituania & 0,24 & 0,46 & $-0,22$ \\
\hline Portugal & 0,24 & 0,46 & $-0,22$ \\
\hline Letonia & 0,27 & 0,46 & $-0,20$ \\
\hline República- & 0,22 & 0,47 & $-0,26$ \\
\hline Checa & 0,17 & 0,52 & $-0,36$ \\
\hline Grecia & 0,18 & 0,54 & $-0,54$ \\
\hline Slovenia & 0,11 & 0,58 & \\
\hline Slovakia & 0,08 & 0,62 & \\
\hline Hungría & & & \\
\hline & & & \\
\hline
\end{tabular}

Fuente: Elaboración propia 


\section{Resultados según PROMETHEE II}

\section{Ranking completo}

Flujos netos: $\emptyset=\emptyset+-\emptyset$ -

Según el reporte del Decisión Lab (Cuadro $\mathrm{N}^{\circ} 7$, última columna), se observa que las diferencias en general son iguales al hacer el cálculo manual.

En este ranking, como se puede observar, Suecia supera en fortaleza a los demás países con $\emptyset=0,61$, y el más débil es Hungría con $\emptyset=$ $-0,55$; por lo tanto, el que tiene mayor crecimiento sostenible es Suecia, y el de menor crecimiento sostenible es Hungría, lo que coincide con los resultados obtenidos con PROMETHEE I.

\section{Análisis de sensibilidad de los pesos}

Asimismo, el Decision Lab nos reporta en una tabla la sensibilidad de los pesos de cada criterio. Se debe variar solo un peso dentro de su rango especificado y los demás pesos deben mantenerse fijos: Por ejemplo, el criterio esperanza de vida sana a partir de los 65 años, tiene un peso igual a 0,1460 y el mínimo valor que puede tomar es de 0,1317 y el máximo es de 0,1540 . Dentro de ese rango, al variar el peso, siempre se obtiene que el ranking completo PROMETHEE II no cambia.

Los criterios como productividad de los recursos (criterio 2) y la esperanza de vida sana a partir de los 65 años (criterio 5) son los más sensibles a los posibles cambios de pesos, y en el otro extremo estarían crecimiento del PBI per cápita (criterio 1), tasa de riesgo de exclusión social (criterio 3) y tasa de empleo de trabajadores mayores (criterio 4) constituyendo el conjunto de criterios menos sensibles.

\section{Resultados según el Plano GAIA}

También mostramos el Plano GAIA (Figura $\mathrm{N}^{\circ} 8$ ), como complemento a la metodología PROMETHEE.

Por ello, se pueden diferenciar varios grupos dentro de la gráfica GAIA. Por ejemplo, los países mejor situados en este ranking de desarrollo sostenible están ubicados en el cuadrante derecho inferior, donde podemos observar a Alemania, Dinamarca, Finlandia y Suecia, países que priorizan la protección del medio ambiente y reciclaje.

Así también se puede observar a Hungría liderando el grupo de los más débiles, país con menos desarrollo sostenible, a la izquierda en el segundo cuadrante.

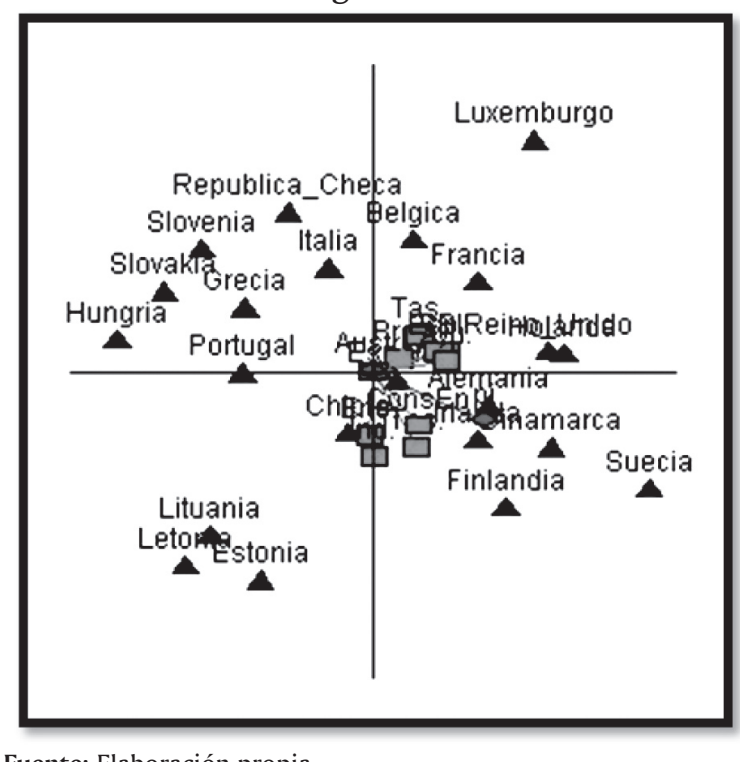

Comparando el comportamiento de los criterios para Suecia y para Hungría que son los menos sostenibles (ver Figura $\mathrm{N}^{\circ}$ ), donde se muestran los indicadores más relevantes que ubican a Suecia como el país más sostenible aún cuando la productividad tiene una puntuación muy baja respecto a los demás indicadores, este indicador tiene como valor 192,13 para Suecia, recordando que el valor máximo es de 1038,8 (ver Cuadro $\mathrm{N}^{\circ} 3$ ) y corresponde a Alemania. Asimismo, podemos obtener gráficos de indicadores para cada una de las alternativas y compararlos dos a dos.

Figura $\mathrm{N}^{\circ} 9$ Comparación del comportamiento de los criterios para Suecia y para Hungría

Figura $\mathrm{N}^{\circ} 9$



Fuente: Elaboración propia 


\section{CONCLUSIONES}

La metodología PROMETHEE es una técnica de ayuda sumamente valiosa en un problema de decisión multicriterio al que se enfrenta. Es especialmente apropiado para asistir a un grupo de decisores expertos a alcanzar un consenso sobre un conjunto de alternativas factibles.

La utilización de la modelización multicriterio es una herramienta eficaz en la simulación de clasificaciones de alternativas. Su relativa sencillez, en comparación con otros métodos, la convierte en una metodología a ser considerada en los casos en que se desee modelizar la toma de decisiones en un determinado escenario.

En la elección de los criterios de las alternativas se deben considerar, en general, los más importantes para el caso en estudio; de esta forma, se evita la complejidad del modelo y el costo de tiempo.

En la modelización realizada, las funciones de utilidad de cada criterio se eligen de acuerdo al conocimiento del caso, la intuición y la experiencia considerando la naturaleza de la alternativa (Sánchez, 2007, p.24). Los principales criterios que afectarán a la toma de decisiones es el que mayor o menor peso se asigne, el criterio de menor peso es el menos importante pero relevante para el proceso del estudio.

Es muy importante que las preferencias del decisor encuentren un modelo matemático adecuado que las exprese sin cambiarlas, intentando recoger todos y cada uno de los elementos que tenga establecido el decisor. Se podrían definir otras funciones de preferencias y asociarlas a los criterios originales, esta tarea debe efectuarse cuidadosamente para no distorsionar los resultados finales sino para que reflejen la intensidad de las preferencias del decisor.

Esta técnica puede extenderse a la utilización del análisis clúster donde se plantean simulaciones de alternativas homógeneas, que reducen la dificultad en simular las decisiones de alternativas frecuentemente complejas e influidas por diversos factores.

\section{LITERATURA CITADA}

European Commission. (2006). "Council of the european unión”. Fecha de consulta: 30/05/2013. En: <http://register.consilium.europa.eu/pdf/ en/06/st10/st10917.en06.pdf>.
Eurostat. (2012). Desarrollo sostenible en la Unión Europea: Informe de seguimiento de 2011 sobre la Estrategia de Desarrollo Sostenible de la UE. Oficina de Publicaciones de la Unión Europea, Luxemburgo.

Eurostat. (2011). Sustainable development indicators > Indicators. Oficina Estadística de la Comisión Europea. Luxemburgo. Fecha de consulta: 08/08/2013. En: <http://epp.eurostat. ec.europa.eu/portal/page/portal/sdi/indicators $>$.

Fernández B., Gabriela M. (2002). "Los métodos PROMETHEE: una metodología de ayuda a la toma de decisiones multicriterio discretas". En:Rect@, Revista Electrónica de Comunicaciones y Trabajos de ASEPUMA. (2002). Volumen monográfico. Asociación Española de Profesores Universitarios de Matemáticas para la Economía y la Empresa (ASEPUMA), España. Fecha de consulta: 12/08/2013. Disponible en: < http:// www.uv.es/asepuma/recta/extraordinarios/ Vol_01/01t.pdf>.

Fernández B., G. M. \& Escribano R., M. C. (2006). "Nuevos criterios generalizados para modelar las preferencias del decisor en los métodos de relaciones de superación”. En: Rect@,Revista Electrónica de Comunicaciones y Trabajos de ASEPUMA. (1999). Vol. 7. Asociación Española de Profesores Universitarios de Matemáticas para la Economía y la Empresa (ASEPUMA), España. Fecha de consulta: 20/06/2013. Disponible en: < http://www.uv.es/asepuma/recta/ ordinarios/7/7.3.pdf $>$.

Fernández B., G. M., García C., M. C. \& Escribano R., M. C. (2009). "Métodos de ayuda a la decisión multicriterio con nuevos criterios generalizados: una aplicación a los mercados financieros”. En: Rect@, Revista Electrónica de Comunicaciones y Trabajos de ASEPUMA. (2009). Vol. 17(1). Asociación Española de Profesores Universitarios de Matemáticas para la Economía y la Empresa (ASEPUMA), España. Fecha de consulta: 10/07/2013. Disponible en: Disponible en: <http://www.uv.es/asepuma/ XVII/107.pdf $>$.

Guerrero G., C. (2007). Un modelo multicriterio de localización de centros no deseados con pesos. Publicaciones Consejo Económico y Social de Andalucía (CES-A), Sevilla. 
Franco-Maass, S.; Osorio-García, M.; Nava-Bernal, G. y Regil-García, H. H. (2013). Evaluación multicriterio de los recursos turísticos. Parque Nacional Nevado de Toluca - México. Centro de Investigaciones y Estudios Turísticos, Toluca. ISSN 1851-1732.

Mareschal, B.; Brans, J.P. (1988). "Geometrical representation for MCDM, the GAIA procedure". En: European Journal of Operational Research, $\mathrm{N}^{\circ} 34$.

Pujol P., J. (2002). Un análisis multicriterio del impacto del nuevo marco normativo del agua en los regadíos de la zona regable del Bajo Ter y del Muga (Girona). Tesis Doctoral en Ingenie- ría Agronóma. Departamento de Economía, Sociología y Política Agrarias. Universidad de Córdoba.

Romero, C. (1996). Análisis de las Decisiones Multicriterio. Edit. Isdefe, Madrid.

Sala R., M. (2000). "Aproximación multicriterio a la estructura industrial de la economía española”. En: Economía Industrial. N³32, Vol. II, Lérida.

Sánchez S., P.J. (2007). Modelos para la combinación de preferencias en toma de decisiones: Herramientas y aplicaciones. Tesis Doctoral en Ingeniería Informática. Departamento de Ciencias de la Computación e Inteligencia Artificial. Universidad de Granada. 